\title{
Mechanics of Intercostal Space and Actions of External and Internal Intercostal Muscles
}

\author{
André De Troyer, Suzanne Kelly, Peter T. Macklem, and Walter A. Zin \\ Respiratory Research Unit and Chest Service, Erasme University Hospital, Brussels School of Medicine, Brussels 1070, Belgium; and \\ Meakins-Christie Laboratories, McGill University Clinic, Royal Victoria Hospital, Montreal, Quebec H3A 2B4, Canada
}

\begin{abstract}
It is conventionally considered that because of their fiber orientations, the external intercostal muscles elevate the ribs, whereas the internal interosseous intercostals lower the ribs. The mechanical action of the intercostal muscles, however, has never been studied directly, and the electromyographic observations supporting this conventional thinking must be interpreted with caution. In the present studies, the external and internal interosseous intercostal muscles have been separately stimulated in different interspaces at, above, and below end-expiratory rib cage volume in anesthetized dogs. The axial (cephalo-caudal) displacements of the ribs were measured using linear displacement transducers. The results indicate that when contracting in a single interspace and other muscles are relaxed, both the external and internal intercostals have a net rib elevating action at end-expiratory rib cage volume. This action increases as rib cage volume decreases, but it progressively decreases as rib cage volume increases such that at high rib cage volumes, both the external and internal intercostals lower the ribs. Stimulating the intercostal muscles in three adjacent intercostal spaces simultaneously produced similar directional rib motion results. We conclude that $(a)$ in contrast with the conventional thinking, the external and internal interosseous intercostals acting alone have by and large a similar effect on the ribs into which they insert; (b) this effect is very much dependent on rib cage (lung) volume; and (c) intercostal muscle action is primarily determined by the resistance of the upper ribs to caudad displacement relative to the resistance of the lower ribs to cephalad displacement. The lateral intercostals, however, might be more involved in postural movements than in respiration. Their primary involvement in rotations of the trunk might account for the presence of two differently oriented muscle layers between the ribs.
\end{abstract}

\section{Introduction}

The action of the external and internal intercostal muscles on the ribs has been the subject of considerable controversy throughout medical history, and up to the middle of this century the most varying and opposite points of view have had strong supporters among physiologists (1-5). The most widely held view at present is that associated with the name

Address reprint requests to Dr. De Troyer, Chest Service, Erasme University Hospital, 808, Route De Lennik, 1070 Brussels, Belgium.

Received for publication 18 April 1984 and in revised form 31 October 1984

J. Clin. Invest.

(c) The American Society for Clinical Investigation, Inc.

0021-9738/85/03/850/08 $\$ 1.00$

Volume 75, March 1985, 850-857 of Hamberger (6). This theory, inferred from the orientations of intercostal muscle fibers and the distances between their costal insertions and the center of rotation of the ribs, maintains that the external intercostals are inspiratory in their action on the rib cage and the internal are expiratory, with the exception of the interchondral portion ("the parasternals"), which is inspiratory. Indeed, many electromyographic studies of intercostal muscles have reported a phasic behavior of these muscles in accord with this theory (7-10). The present studies, however, provide experimental evidence that the external and internal interosseous intercostals, when they act alone, have a similar effect on the ribs, and further that this effect is very much dependent on lung (rib cage) volume. We begin by analyzing the theory of Hamberger.

Hamberger's theory. This theory is illustrated by the diagram shown in Fig. 1. Hamberger maintained that, as the fibers of the external intercostals slope obliquely caudad and ventrally from the rib above to the one below, their lower insertion is more distant from the center of rotation of the ribs than the upper one (Fig. 1, top). Hence, when the muscle contracts with its force equal and opposite at both insertions, the torque acting on the lower rib, tending to raise it, is greater than that acting on the upper rib, tending to lower it. The net effect of contraction of the external intercostal would therefore be to raise the ribs into which it inserts. On the other hand, the fibers of the internal intercostals slope obliquely caudad and dorsally from the rib above to the one below, so that their lower insertion is less distant from the center of rotation of the ribs than the upper one (Fig. 1, bottom). As a result, when the muscle contracts, the torque acting on the lower rib is smaller than that acting on the upper rib and hence, the net effect of contraction of the internal intercostal would be to lower the ribs to which it is attached. The internal intercartilaginous intercostals (the "parasternals") have the same fiber orientation as the internal interosseous intercostals, but according to Hamberger their action should be referred to the sternum, not to the spine. Indeed, these muscles originate from the lateral margin of the sternum and insert into the superior surface of the costal cartilages. To the extent that the geometric relationship between the parasternals and the sternum is similar to that between the external intercostals and the spine, the parasternals should thus raise the ribs when they contract. Thus, in view of the fiber orientations and insertions, the external intercostals and the parasternals would elevate the ribs and hence, would be inspiratory for the rib cage, whereas the internal interosseous intercostals would lower the ribs and therefore act to deflate the rib cage. It must be stressed, however, that although these ideas have prevailed for the past $25 \mathrm{yr}$, they have never been demonstrated experimentally, and their only support, as discussed below, must be interpreted with caution. 
External

Intercostal

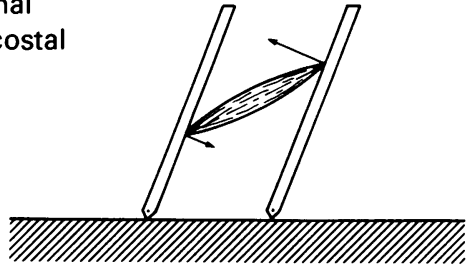

Internal

Intercostal



Figure 1. Diagram illustrating Hamberger's theory. In each panel, the hatched area represents the spine (head on the left) and the two bars oriented obliquely represent two adjacent ribs. The intercostal muscles are depicted as single bundles, and the torques acting on the ribs during intercostal muscle contraction are pictured by arrows. See text for further explanation.

The hypothesis that the parasternals are inspiratory in their action on the rib cage has been recently confirmed by experiments in dogs, which have shown that these muscles indeed elevate the ribs into which they insert by decreasing the angle between the lateral border of the sternum and the upper border of the ribs (11). On the other hand, the prediction that the external and internal interosseous intercostals act in opposing directions on the ribs has not been confirmed experimentally. In fact, we have recently observed that when either the external or internal interosseous intercostal of a given interspace is stimulated at end-expiratory lung volume, both muscles have a net elevating action on the ribs to which they are attached (12). These findings, supporting the previous observation by Duchenne (2) in a man in whom stimulation of the external intercostal with or without the internal intercostal in one interspace always elevated the lower rib, thus suggested that the muscle fiber orientation is not the primary determinant of the mechanical action of the lateral intercostals. The further observation that at functional residual capacity (FRC) ${ }^{1}$, when the rib cage is below its neutral position, its inherent elastic recoil tends to move it cephalad, was interpreted to indicate that lateral intercostal muscle action is in fact primarily determined by the relative resistance of the ribs to upward and downward displacement (12). The experiments reported here were designed to investigate this hypothesis more systematically.

Rationale. The following reasoning prompted us to examine how the actions of the external and internal interosseous intercostals are affected by changes in lung (rib cage) volume. If Hamberger's theory were correct (that is, if the orientations and insertions of the intercostal muscle fibers determined the actions of these muscles on the ribs), one would expect the direction of action of the lateral intercostal muscles to be

1. Abbreviations used in this paper: EMG, electromyographic; FRC, functional residual capacity; TLC, total lung capacity; Zcaud, the impedance of the caudad rib to upward motion; Zceph, the impedance of the cephalad rib to downward motion. independent of volume. The external intercostals would always act to elevate the ribs and the internal would always act to lower the ribs. In contrast, if the actions of the lateral intercostals in a given interspace were primarily determined by the impedance of the cephalad rib to downward motion (Zceph) relative to the impedance of the caudad rib to upward motion (Zcaud), they might be profoundly affected by changes in volume. The rib cage is indeed below its neutral position at FRC but above it at total lung capacity (TLC) (13). Furthermore, Zceph should be affected by passive tension in the neck muscles (scalenes and sternocleidomastoids), and thus increase as lung volume decreases and these muscles are stretched; whereas Zcaud might increase at high lung volumes when the neck muscles are shortened and the abdominal muscles are stretched. Under these circumstances, contraction of either the external or internal interosseous intercostals might elevate the rib cage at low volumes (when Zceph is greater than Zcaud) and lower the rib cage at high volumes (when Zceph is less than Zcaud).

\section{Methods}

The experiments were performed on nine supine adult dogs (body weight $16-23 \mathrm{~kg}$ ) under pentobarbital sodium (Nembutal) anesthesia (initial dose, $25 \mathrm{mg} / \mathrm{kg}$ i.v.). A tracheostomy was performed, and a venous cannula was inserted to give maintenance doses of anesthetic. The level of anesthesia was regulated to keep the corneal reflex abolished throughout the experiment.

The rib cage and the intercostal muscles were exposed from the first to the tenth rib by deflection of the skin and the consecutive layers of muscles. Hooks were then screwed into two adjacent ribs on the anterior or midaxillary line and connected by inextensible threads to linear displacement transducers positioned along the animal's longitudinal body axis to measure the axial displacements of the ribs (Fig. 2). In five animals, a hook was also screwed into the sternum at the level of the fourth or fifth intercostal space and connected to an additional displacement transducer to measure the axial displacement of the sternum. As described in detail previously (11), the threads were passed over appropriately positioned pulleys to translate the cephalad and caudad motions of the ribs and sternum into up-and-down motions of the cores of the transducers. Gains were adjusted to give the same pen deflection in the three channels for a given, known displacement of the core. A pair of stimulating electrodes spaced $\sim 2$ $\mathrm{cm}$ apart was then inserted in the midaxillary line superficially in the fibers of the external intercostal muscle connecting the two ribs. These electrodes were silver hooks insulated with polyethylene tubing except for their terminal $0.8 \mathrm{~cm}$. The stimulating pulses $(0.2 \mathrm{~ms}$ in duration, $20-100 \mathrm{~Hz}$ in frequency) were adjusted from 5 to $10 \mathrm{~V}$ for maximum effect without activation of the other intercostal muscles of the same interspace. This technique of stimulation has previously been shown to avoid significant activation of the internal intercostal muscle layer (12). The external intercostal was thus stimulated at various lung volumes, at, above, and below FRC obtained by applying positive (up to $30 \mathrm{~cm} \mathrm{H}_{2} \mathrm{O}$ ) or negative (down to $20 \mathrm{~cm} \mathrm{H}_{2} \mathrm{O}$ ) pressure to the tracheostomy tube. After the external intercostal was studied, the muscle was surgically removed and the internal interosseous intercostal was exposed and stimulated at the same different lung volumes. Studies were made on 19 intercostal spaces ranging between the third and the eighth. In eight experiments on five animals, we also studied the effect of simultaneous stimulation of three adjacent (external or internal) intercostal muscles at both low and high lung volumes.

We also measured airflow at the tracheostomy tube with a Fleish pneumotachograph and a differential pressure transducer (Validyne Engineering Corp., Northridge, CA) during the stimulations at FRC, and airway pressure with a pressure transducer connected to the tube during the stimulations at higher or lower volumes. All measurements were obtained during apnea induced by mechanical hyperventilation. 


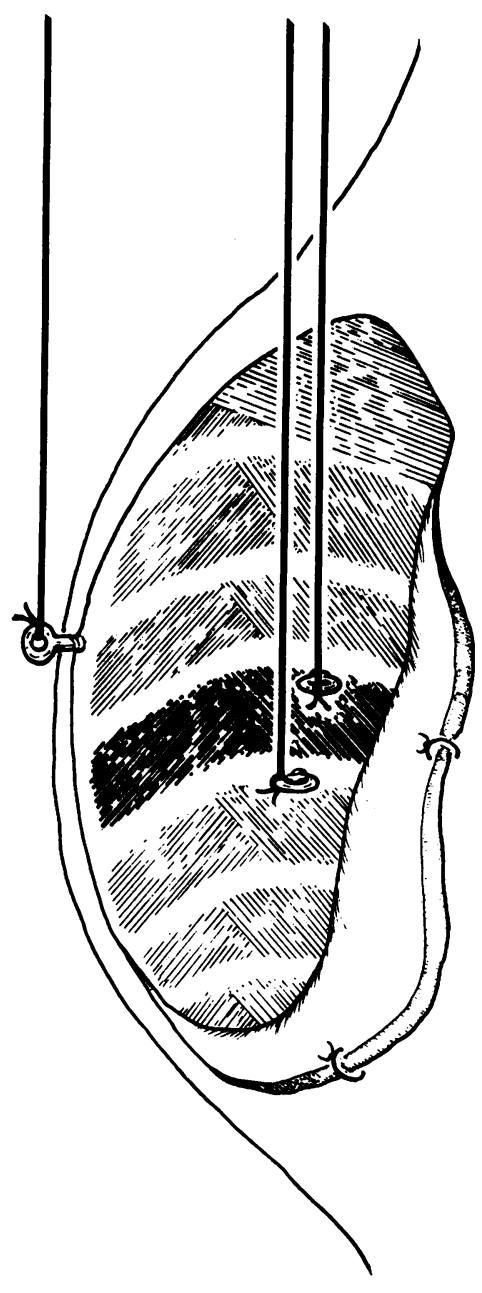

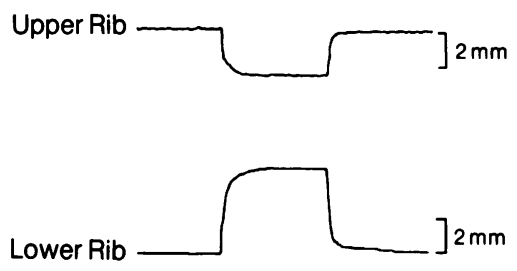

$1 \mathrm{~s}$.

Figure 3. Effect of stimulating the external intercostal muscle in one interspace at FRC on the axial displacements of the ribs situated immediately above (top) and below (bottom). In the two channels, upward deflections indicate a cephalad displacement, and downward deflections the reverse.

(Fig. 4, left). There was no systematic difference between the interspaces situated in the cranial and caudad portions of the rib cage. Stimulating the internal interosseous intercostal in one interspace at FRC gave similar rib motion results. Here also, no matter which interspace was studied, the cephalad displacement of the rib below was about twice as large as the caudad displacement of the rib above (Fig. 4, right). Thus, this confirms our previous observation (12) that when the internal interosseous intercostal muscle contracts alone in a single interspace, the net action of the muscle at FRC is also inspiratory for the ribs to which it is attached.

Increasing lung (rib cage) volume above FRC profoundly

Figure 2. Close-up view of the rib cage preparation. The animal's head is on the left and its abdomen on the right side of the rib cage.
Finally, in five of the animals, we also measured the stress-strain relation of the ribs (with their anatomic attachments) along the cephalo-caudal axis of the rib cage. The technique has been previously described (12). While the animal was apneic, weights $(100-500 \mathrm{~g})$ were applied to one rib, first in the cephalad and then in the caudad direction. The resulting axial displacements of the rib were measured with a linear displacement transducer placed, here too, along the longitudinal body axis of the animal. Ranging between the third and the seventh rib, 11 ribs were studied. For each rib examined, the procedure was done at FRC and at the higher volumes at which the intercostal muscles had been previously stimulated. At each volume studied, each weight was applied to the rib at least three times, both in the cephalad and the caudal direction. Data presented are thus averages of at least three measurements at each volume and in each direction.

\section{Results}

Muscle stimulation experiments. A representative example of rib motions produced by stimulation of the external intercostal muscle in one interspace at FRC is shown in Fig. 3. Stimulation of the external intercostal muscle always caused a cephalad displacement of the rib below and a caudad displacement of the rib above. The cephalad displacement of the rib below, however, was twice as large as the caudad displacement of the rib above, thus indicating that for the ribs into which it inserts, the net effect of contraction of the external intercostal muscle is inspiratory. Almost identical records were obtained during external intercostal stimulation in the 19 interspaces investigated influenced the rib displacements produced by the external and internal intercostals. Representative records are shown in Fig. 5. As lung volume increased, the cephalad displacement of the lower rib due to external intercostal stimulation gradually decreased, whereas at the same time, the caudad displacement of the upper rib progressively increased (Fig. $5 \mathrm{~A}$ ). As a result, at the volume corresponding to $10 \mathrm{~cm} \mathrm{H}_{2} \mathrm{O}$ positive airway pressure, although the cephalad displacement of the lower rib was still slightly larger than the caudad displacement of the upper rib, the motions of the two ribs making up the intercostal

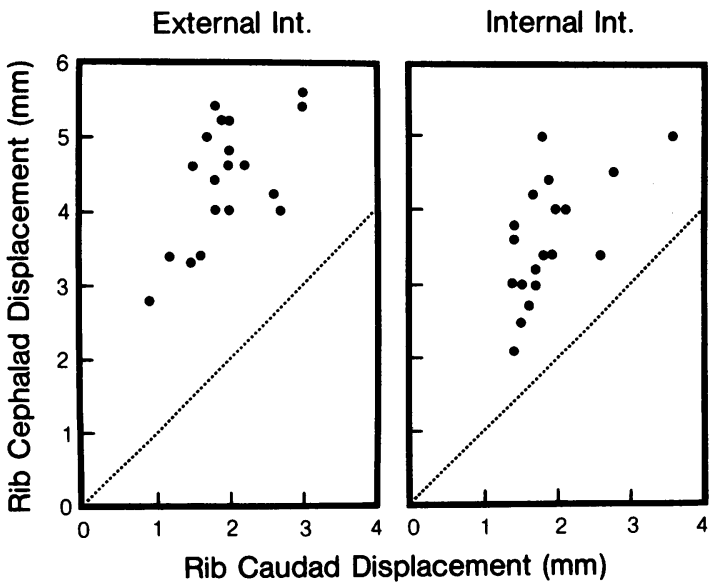

Figure 4. Relative axial displacements of the upper ( $\mathrm{X}$ axis) and lower ( $\mathrm{Y}$ axis) ribs during separate stimulation of the external (left) and internal interosseous (right) intercostal muscles in one intercostal space at FRC. Results were obtained in 19 interspaces. The broken line in each panel is the identity line. Note that in all interspaces, the cephalad displacement of the rib below was greater than the caudad displacement of the rib above. 


\section{A. External Intercostal}

$$
\text { FRC } \quad 10 \mathrm{~cm} \mathrm{H}_{2} \mathrm{O} \text { PEEP } 20 \mathrm{~cm} \mathrm{H}_{2} \text { O PEEP }
$$

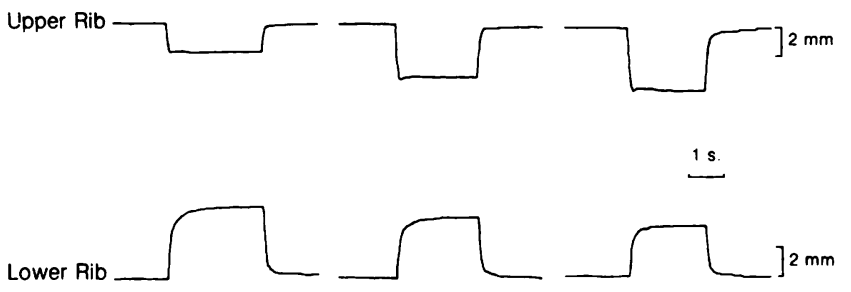

\section{B. Internal Intercostal}

$$
\text { FRC } \quad 10 \mathrm{~cm} \mathrm{H}_{2} \text { O PEEP } \quad 20 \mathrm{~cm} \mathrm{H}_{2} \text { O PEEP }
$$
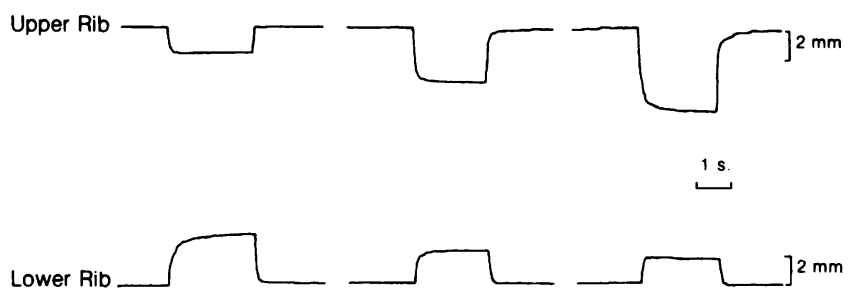

Figure 5. Effect of stimulating separately the external $(A)$ and the internal interosseous $(B)$ intercostal muscle in one intercostal space at different rib cage volumes at and above end-expiratory lung volume. Same legend as Fig. 3.

space were approximately equal in amplitude. Increasing lung volume further amplified this phenomenon, and when $20 \mathrm{~cm}$ $\mathrm{H}_{2} \mathrm{O}$ positive pressure was applied at the airway opening, the cephalad displacement of the lower rib was clearly smaller than the caudad displacement of the upper rib. At high volumes, the net effect of contraction of the external intercostal is therefore to lower the ribs into which it inserts.

As illustrated in Fig. $5 \mathrm{~B}$, increasing lung volume above FRC had a qualitatively similar effect on the rib motions produced by internal interosseous intercostal stimulation. The records shown in this figure were obtained during stimulation of the internal intercostal corresponding to the external intercostal shown in Fig. $5 \mathrm{~A}$. Here also, the net elevating effect on the ribs progressively decreased as volume increased, and it was eventually reversed into a net lowering effect at high volumes. Note, however, that during internal intercostal stimulation, in contrast with external intercostal stimulation, the cephalad displacement of the lower rib was already slightly smaller than the caudad displacement of the upper rib at the volume corresponding to $10 \mathrm{~cm} \mathrm{H}_{2} \mathrm{O}$ positive airway pressure. Also, the net rib lowering effect of the internal intercostal at $20 \mathrm{~cm} \mathrm{H}_{2} \mathrm{O}$ positive airway pressure was greater than that of the external intercostal. Fig. 6 summarizes the rib motion results obtained in the 19 interspaces investigated. All the interspaces of the nine animals behaved similarly. The only interanimal difference that was seen related to the magnitude of the airway pressure needed to reverse the net rib elevating action into a net lowering one. That is, in some animals, 7.5 $\mathrm{cm} \mathrm{H}_{2} \mathrm{O}$ positive airway pressure was sufficient to produce the reversal, whereas in others, $20 \mathrm{~cm} \mathrm{H}_{2} \mathrm{O}$ positive airway pressure was required to do so.

Decreasing volume below end-expiratory lung volume had the opposite effect, as illustrated in Fig. 7 for external intercostal stimulation. For the same muscle stimulation, applying a

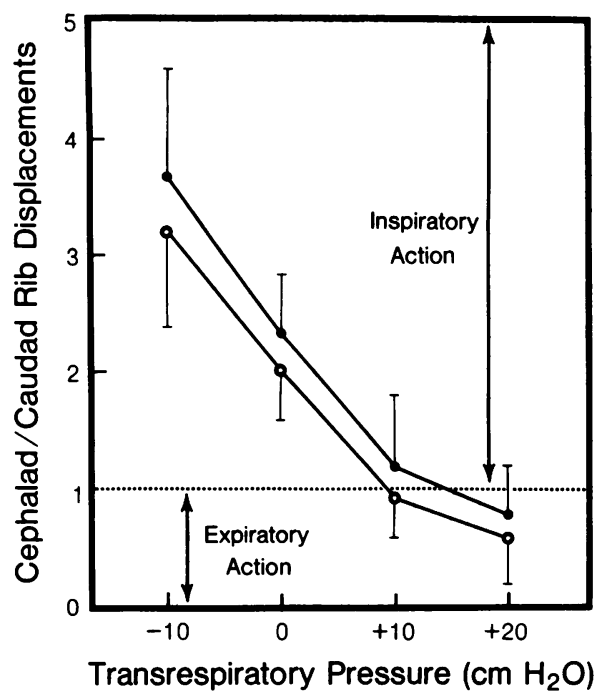

Figure 6. Effect of lung volume on the actions of the external (closed circles) and internal (open circles) intercostal muscles on the ribs into which they insert. Represented are the average data of 19 interspaces in nine animals. The muscle actions are expressed as the ratio of cephalad displacement of the rib below to caudad displacement of the rib above. This ratio is thus $>1$ when the cephalad displacement of the rib below is greater than the caudad displacement of the rib above (inspiratory action), while it is $<1$ when the caudad displacement of the rib above is greater than the cephalad displacement of the rib below (expiratory action). The bars represent \pm 1 SD.

negative pressure at the airway opening increased the cephalad displacement of the lower rib and decreased the caudad displacement of the upper rib. This was true for all the interspaces investigated during both external and internal intercostal stimulation (Fig. 6).

When external or internal intercostal stimulation caused the sternum to be displaced axially a significant amount, the motion was usually cephalad in direction during external intercostal stimulation, and caudad during internal intercostal stimulation. These displacements, however, were always small ( $<0.5 \mathrm{~mm})$ in amplitude compared with the concomitant displacements of the ribs, and in most experiments, whatever the volume, they could not be detected.

The flow rate and airway pressure measurements obtained during the stimulations were by and large consistent with the rib motion results, a net rib elevating action usually associated

FRC $10 \mathrm{~cm} \mathrm{H}{ }_{2} \mathrm{O}$ NEEP

Upper Rib
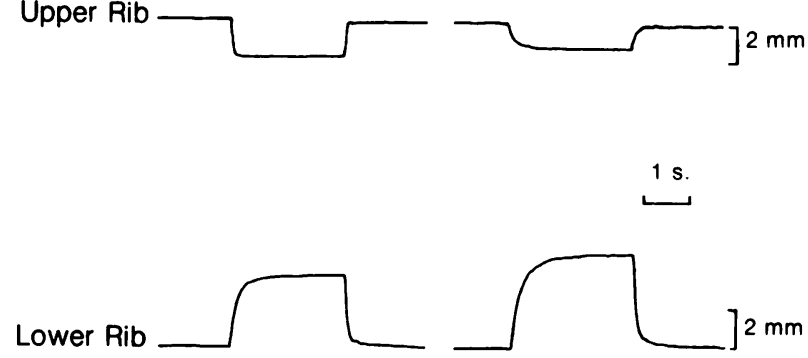

Figure 7. Effect of stimulating the external intercostal muscle in one intercostal space at and below end-expiratory rib cage volume. Same legend as Fig. 3. 
with inspiratory flow rate and negative airway pressure and vice-versa. The only exceptions to this rule were seen during stimulation of the lowermost interspaces at or below FRC and during stimulation of the uppermost interspaces at high volumes. For example, during external or internal intercostal stimulation in the eighth interspace at FRC, the net rib elevating action was associated with a small expiratory flow rate or no flow rate at all, presumably because the elevation of the last two or three ribs was not sufficient to compensate for the mild lowering of the first eight ribs. For a presumably similar reason, airway pressure often remained stable during stimulation of the external or internal intercostal muscle in the third interspace at high volumes.

Nevertheless, in the eight experiments in which the external and internal interosseous intercostals were stimulated at or below FRC in three adjacent interspaces simultaneously, the two ribs situated in between moved in a cephalad direction and an inspiratory flow rate or a negative airway pressure was recorded (Fig. 8). Stimulating the external or internal intercostals in 3 adjacent interspaces simultaneously at high lung volumes always resulted in a caudad displacement of the two ribs in between, and positive airway pressure. These results were obtained whether the interspaces examined were in the cranial (second, third, and fourth), middle (fourth, fifth, and sixth), or caudal (sixth, seventh, and eighth) portion of the rib cage.

Stress-strain relation of the ribs. For any given rib and at any rib cage volume, no matter which weight was applied, the measurements of rib axial displacements were very reproducible. On three separate determinations, the variability of the rib motion results was always $<10 \%$. Applying weights to the ribs at FRC in the cephalad or the caudad direction caused them to be displaced axially in proportion to the weight applied; the greater the weight, the larger the axial displacement of the rib. As illustrated in Fig. 9 (left), however, the displacements observed were not linearly related to the weights applied, and also, they were different depending on whether the weights were applied in the cephalad or the caudad direction. For any given weight examined, the rib at FRC was displaced substantially more in the cephalad than in the caudad direction. The slope of the curves thus obtained represents the strain-stress

\section{External Intercostals Internal Intercostals}
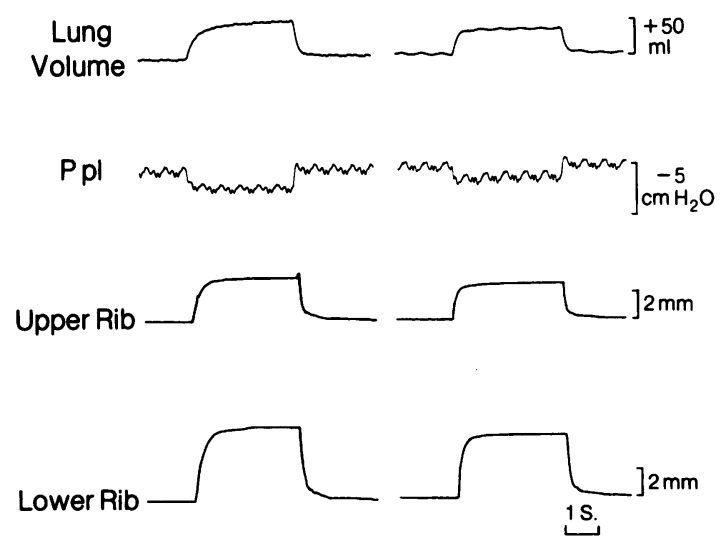

Figure 8. Effect of stimulating the external (left) or internal (right) intercostals in three adjacent interspaces simultaneously at FRC on the axial displacements of the two ribs situated in between. The associated changes in lung volume and pleural pressure (Ppl) are shown. Same conventions as Fig. 3.

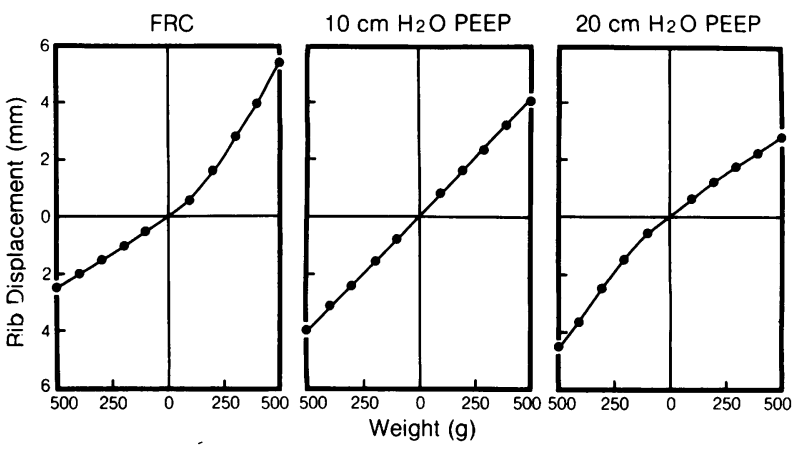

Figure 9. Stress-strain relation of one rib at different rib cage volumes at and above end-expiratory volume. This relation was obtained by applying weights $(100-500 \mathrm{~g})$ to the rib successively in the cephalad (upper portion of the curves) and caudad (lower portion) direction. The rib shown here corresponds to the lower rib of Fig. 5. Note that the relationship is alinear and that the rib's tendency to move cephalad decreases as rib cage volume increases, while at the same time, the rib's tendency to move caudad increases.

relationship of the rib cage at a given point in the cephalad or caudad direction, which can be expressed as millimeters of displacement per kilogram of weight. In the present context, by analogy with the more conventional measurements of static elastic properties of the respiratory system, we found it convenient to adopt the terms cephalad and caudad rib compliance, respectively. Because of the nonlinearity of the stress-strain characteristic, the cephalad rib compliance at FRC was thus greater than the caudad rib compliance. All 11 ribs examined behaved similarly, and although in each animal the cephalad and caudad rib compliance progressively increased when going from the cranial to the caudal portion of the rib cage, the cephalad/caudad rib compliance ratio was not affected by the location of the rib. For the 11 ribs investigated, the cephalad/ caudad rib compliance ratio at FRC averaged $1.84 \pm 0.42$ (mean \pm SD).

This pattern was also markedly affected by increases in lung (rib cage) volume (Fig. 9). As volume increased above FRC, the weight-induced displacements of the rib gradually decreased in the cephalad direction but increased in the caudad direction. That is, the cephalad rib compliance declined while the caudad rib compliance increased as rib cage volume increased. In the case illustrated, the cephalad and caudad rib compliance were equal at the volume corresponding to $10 \mathrm{~cm}$ $\mathrm{H}_{2} \mathrm{O}$ positive airway pressure. When volume increased further, the cephalad rib compliance became smaller than the caudad compliance. As shown in Fig. 10, all 11 ribs examined behaved similarly, the average (mean $\pm \mathrm{SD}$ ) cephalad/caudad compliance ratio being $1.09 \pm 0.13$ and $0.78 \pm 0.16$ at 10 and $20 \mathrm{~cm} \mathrm{H}_{2} \mathrm{O}$ positive airway pressure, respectively.

\section{Discussion}

The findings that the external and internal interosseous intercostal muscles have in general a similar effect on the ribs and that this effect varies considerably as a function of rib cage volume strongly oppose the conventional thinking that the externals are inspiratory and the internals are expiratory in their action on the ribs. This conventional view, however, apart from its theoretical basis (Hamberger's theory), rests exclusively on selected electrical recordings from intercostal nerves and muscles. One of the studies that has provided the 


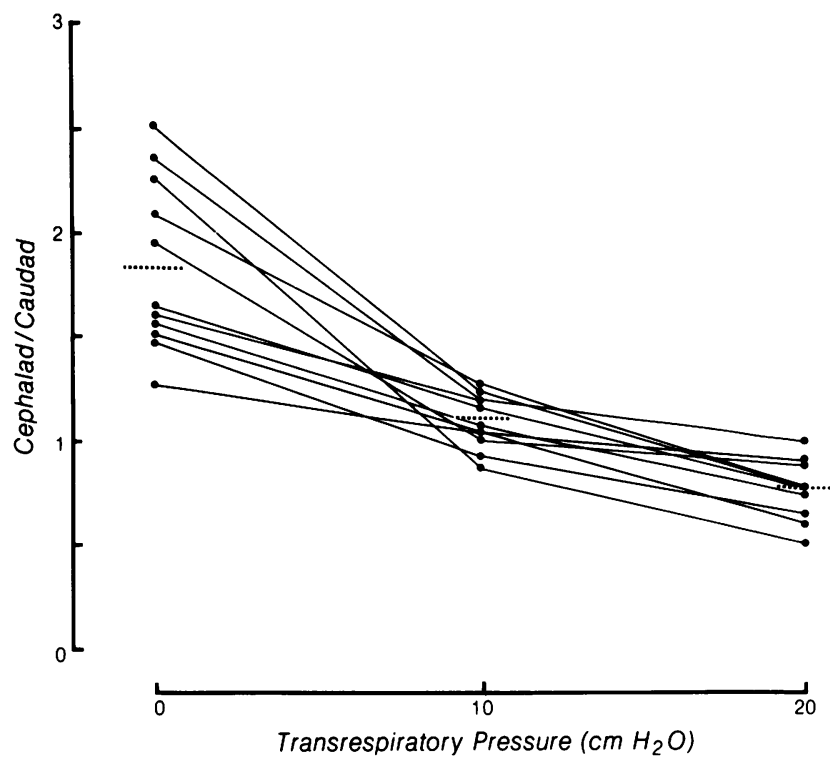

Figure 10. Results of stress-strain measurements in 11 ribs at three different rib cage volumes. Each point represents the ratio of cephalad vs. caudad compliance of one rib at one rib cage volume. The small horizontal dotted lines are mean values. Statistical analysis shows that the differences in cephalad/caudad ratio between 0 and 10 and between 10 and $20 \mathrm{~cm} \mathrm{H}_{2} \mathrm{O}$ transrespiratory pressure are both significant $(P<0.001)$.

basis for conventional thinking in the area is that by Bronk and Ferguson (7), who, recording electrical activity from motor nerves supplying the intercostal muscles in anesthetized or decerebrate cats, reported that the usual picture was a reciprocal alternating discharge of nerve impulses to the external intercostal muscles during inspiration and to the interosseous portion of the internal intercostal muscles during expiration. Other studies supporting the conventional view of intercostal muscles actions are those by Taylor (8), Delhez (10), and Draper et al. (9). These investigators, inserting concentric needle electrodes in the intercostal muscles in awake humans, also observed that the external intercostals only contracted during inspiration, whereas the internal interosseous intercostals were electrically active during expiration.

We believe, however, that these observations in no way determine the mechanical actions of the intercostal muscles, and we justify this on the basis of two arguments. First, motions are frequently complex, requiring contraction not only of agonists but also of synergists, fixators, and even antagonists; and recording electrical activity in a particular muscle during a particular motion does not prove that the muscle is the agonist. This seems to be particularly true in motions as complex as those involved in respiratory acts, as illustrated by the recent observation that the diaphragm is electrically active during forced expiration in humans (14). Although the purpose served by this diaphragmatic electrical activity is uncertain, it is clearly not to aid the abdominal muscles in deflating the lungs. Thus, the observation that one intercostal muscle is electrically active during inspiration and that this contraction is associated with an enlargement of the rib cage does not prove at all that this intercostal muscle is inspiratory in function (that is, causes pleural pressure to fall), nor does it prove that the action of this muscle is to raise the ribs into which it inserts. The ribs could be elevated by other muscles (i.e., scalenes or diaphragm), and the electrical activity observed in the intercostals might be fixating or antagonistic. The argument applies equally to intercostal expiratory EMG activity in relation to rib cage deflation. Thus, as a rule, electromyographic (EMG) recordings can be reliably interpreted only if the mechanical actions of the muscles examined have been previously assessed. Inferring the mechanical action of a muscle from EMG observations alone can lead to absurd conclusions, as dramatically illustrated by the example of the diaphragm during forced expiration.

There is a second reason for which the electrical observations of Taylor $(8)$ and others $(9,10)$ cannot be taken as definitely settling the problem of the actions of the lateral intercostal muscles. The lateral intercostals are two thin muscle layers, only separated by a thin and irregular aponeurotic membrane. Accordingly, unless the rib cage is exposed surgically and the intercostal muscles are carefully dissected, it appears difficult, if not impossible, to ensure that the needle electrodes, even if bipolar, are selectively recording from the individual layers. And indeed, electrical recordings from dissected intercostal muscles in animals have yielded substantially different results from those of Taylor. So, Gesell (4), in anesthetized dogs, and Duron (15), in cats, have reported a very large variability between interspaces and animals with respect to external and internal intercostal EMG activity. In particular, the conventional pattern of externals being active during inspiration and internals being active during expiration was observed only occasionally. In connection with this, it is worth noting that Bronk and Ferguson (7) have also pointed out several individual variations and discordant findings, including a pattern in which the motor nerves to the external and internal intercostal muscles were discharging synchronously during inspiration. Although one may argue that there may be species variations and that observations made in animals cannot be reliably applied to man, note that a similar interindividual variability in intercostal muscle EMG has since been found in humans (L. Delhez, personal communication). On the basis of these considerations, it thus seems that the electrical behavior of the lateral intercostal muscles is more complex and the experimental evidence in support of Hamberger's theory is far weaker than usually thought.

The present studies thus represent the first systematic attempt to establish directly the mechanical actions of the external and internal intercostal muscles on the ribs, and most of the results are in sharp contrast to Hamberger's theory. We found that both the external and internal interosseous intercostals have a net rib elevating action when they contract alone in a single interspace at low lung (rib cage) volumes, that is when the rib cage is below its neutral position and passive tension is presumably present in the neck muscles (Figs. 4 and 7). We also observed that this action is considerably affected by increases in lung volume so that at high volumes, while the rib cage is above its neutral position and the abdominal muscles presumably exert greater passive tension than the shortened neck muscles, both the external and internal intercostals have a net lowering action on the ribs (Figs. 5 and 6). Finally, we found the same directional rib motion results when either the external or internal intercostals were stimulated in three adjacent interspaces simultaneously (Fig. 8). Although the effect of simultaneous contraction of more than three adjacent intercostal muscles has not been studied, the present observations thus suggest that it is the impedance of the cephalad ribs to downward motion (Zceph) relative to the impedance of the caudad ribs to upward motion 
(Zcaud) that primarily determines intercostal muscle action, not the orientation of the muscle fibers (torque effect).

This concept is illustrated by the model shown in Fig. 11. The intercostal space is represented as two bodies (ribs) connected by two muscles (an external and an internal intercostal), and it is suspended between two springs corresponding to Zceph and Zcaud, respectively. In this model, the distance between the two bodies plus the sum of the length of the two springs is constant. As a result, contraction of a muscle causes lengthening of the two springs. In addition, because the force exerted by the muscle is equal and opposite at both insertions, the impedance to motion of each body is determined solely by the elastic properties of the spring to which it is attached. The body attached to the spring with the greatest compliance will move the most, while that attached to the spring with the lowest compliance will move the least. Thus, the relative compliance of each spring will determine whether the net effect of the muscle is an upward or downward displacement of the bodies. At FRC, the external and internal intercostals were found to cause a net upward displacement of the ribs, suggesting that Zceph is greater than Zcaud. Accordingly, the spring corresponding to Zceph in the model is more extended (less compliant) than the one corresponding to Zcaud (Fig. 11,
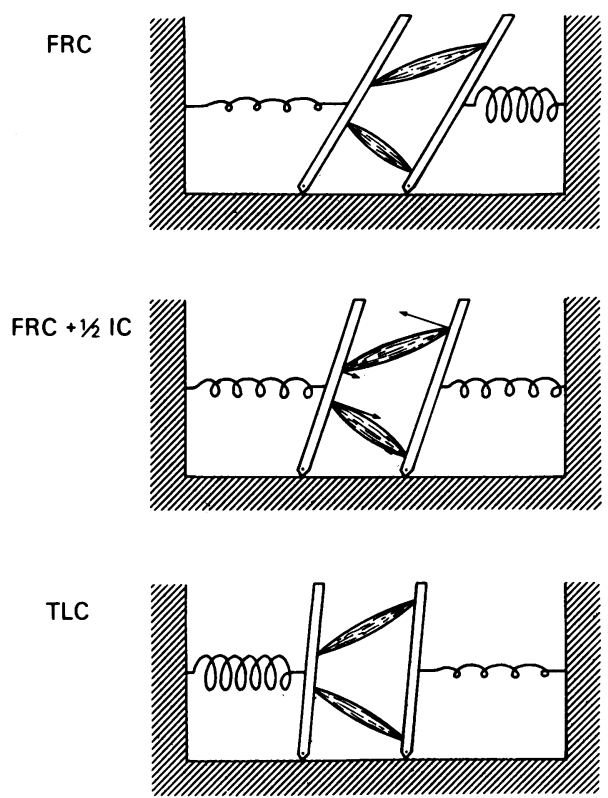

Figure 11. Mechanical model illustrating our concept of intercostal muscle action. In each panel, the horizontal hatched area represents the spine (head on the left), and the two bars oriented obliquely represent two adjacent ribs that are connected by an external and an internal intercostal muscle (As in Fig. 1, no attempt was made here to make a representation of intercostal space that is anatomically accurate; thus, the intercostal muscles are pictured by separate, single bundles, and their fiber orientations are approximate). The spring on the right corresponds to the impedance of the caudad rib to upward motion (Zcaud), and the spring on the left represents the impedance of the cephalad rib to downward motion (Zceph). At end-expiratory rib cage volume (FRC, top), Zcaud is smaller than Zceph. As a result, the external and internal intercostals have a net rib elevating action. By contrast, at high rib cage volumes (TLC, bottom), Zcaud is greater than Zceph. Hence, both the external and internal intercostals have a net rib lowering action. At mid-rib cage volume (FRC $+1 / 2$ IC, middle), Zcaud is equal to Zceph, and the torque effect, as shown by arrows, determines the intercostal muscle action. For further explanation, see text. top). Furthermore, if Zceph and Zcaud remained constant with changes in lung volume (linear systems), the actions of the intercostal muscles on the rib cage would be the same at all lung volumes. The finding that intercostal muscle action is markedly affected by changes in lung volume thus implies that Zceph and Zcaud must change relative to one another. The observed stress-strain relationship of the ribs (Figs. 9 and 10) agrees with this prediction, and by analogy in Fig. 11, the force-length characteristic of each spring is alinear so that at high lung volumes (bottom) the spring corresponding to Zceph is more compressed (more compliant) than the one corresponding to Zcaud.

Two predictions arise from this model. First, it predicts that the net actions of the external and internal intercostals on the ribs are close to zero and go in opposing directions when Zceph is equal to Zcaud. Indeed, in this particular condition, to the extent that the impedance effect is removed, the torque effect should remain the only active component and make the external intercostals elevate the ribs and the internals lower the ribs (Fig. 11, middle). We tested this prediction experimentally and found that it was correct. At mid-lung volume, the external and internal intercostals caused the adjacent ribs to move nearly equally to each other, and the net effect was elevating in the first instance and lowering in the second (Fig. 5).

The second prediction arising from the model is that the actions of the lateral intercostal muscles should be markedly affected by the pattern of activation of the muscles that insert into the extremities of the rib cage and possibly also by the sequence of intercostal muscle recruitment. Active contraction of the neck or abdominal muscles should indeed have a critical influence on Zceph and Zcaud. So, contraction of the neck muscles, by causing cranial fixation of the first two ribs (that is, making Zceph very high), would have the effect of making the lateral (external or internal) intercostals elevate the ribs below, no matter what the intrinsic effect of increasing rib cage volume would be. Conversely, prior contraction of the abdominal muscles, by fixing the lowermost ribs, would have the effect of making the lateral intercostals lower the ribs above, irrespective of the fact that reducing rib cage volume would by itself make them elevate the ribs. Transmitting the traction placed on the uppermost and lowermost ribs to the remaining ribs may thus determine most of the respiratory action of the external and internal intercostals. Intercostal muscle action might be affected by the sequence of intercostal muscle recruitment in a similar fashion. For example, contraction of the external or internal intercostal muscle in the fifth interspace would fix the sixth rib cranially in such a way that subsequent and/or weaker contraction of the intercostals in the sixth interspace would produce an elevation of the ribs below. Conversely, if the intercostals of the sixth interspace contracted first, they would prevent the sixth rib from moving upward and would cause subsequent contraction of the intercostals in the fifth interspace to lower the ribs above. A caudocephalic gradient of muscle activity could thus make the lateral intercostals be expiratory in their action on the rib cage, whereas a cephalocaudal gradient would make the same muscles be inspiratory.

This prediction has not yet been tested, and there are no data of which we are aware that suggest that the mechanical action of the external or internal intercostals varies as a function of the muscles that insert into the extremities of the rib cage or as a function of the sequence of intercostal muscle 
activation. This prediction, however, if correct, would allow many electrical observations to be reconciled with our concept. Indeed, the muscles of the neck are recruited as ventilation increases $(16,17)$ or as one shifts from supine to upright $(10$, 18 ), and at the same time the inspiratory intercostals of the upper interspaces seem to be activated preferentially relative to those of the lower interspaces $(8,19-21)$. Conversely, during expiratory efforts, the abdominal muscles are recruited and intercostal expiratory EMG activity seems to occur along a caudocephalic gradient $(8,22)$. If the neural activation of the external intercostals was coupled with that of the neck inspiratory muscles, and if the neural activation of the internal interosseous intercostals was coupled with that of the abdominals, the externals thus could have the effect of elevating the ribs, whereas the internals would have the effect of lowering the ribs. Despite the mechanical observations reported here, the external and internal intercostals thus may still act on the rib cage in opposing directions during breathing efforts. It is important to realize, however, that this would not result from differences in the intrinsic mechanical actions of these muscles as usually thought, but from differences in the neural patterns of recruitment and coordination.

Whatever the potential differences between the external and internal intercostals in terms of patterns of neural activation, one might wonder why two muscle layers with different fiber orientations exist between the ribs if these muscles have the same intrinsic actions. Would not a single muscle layer suffice to transmit the traction and displace the ribs during breathing? The present observations suggest that it probably would, at least for respiration. It is possible, however, that the intercostal muscles situated laterally in the rib cage are more involved in postural movements than in respiration. Two lines of evidence support this hypothesis. First, studies in cats by Duron (15) have shown that the external and internal interosseous intercostals, unlike the diaphragm and the parasternals, are abundantly supplied with muscle spindles, and most often exhibit tonic electrical activity, unrelated to the phases of respiration, during breathing. Second, it seems likely that the distances between the ribs, while being fairly constant on chest $\mathrm{X}$-ray films during breathing efforts, vary noticeably during rotations and flexions of the trunk. Dramatic illustrations of such changes in distance between the ribs are provided by discus throwers and belly dancers, and although technical factors cannot be excluded with certainty, such postural movements have been reported to elicit substantial EMG activity in the intercostals (23). Accordingly, a hypothesis based on the similarities of the intercostal and abdominal muscles in terms of muscle fiber orientations may be proposed, which would account for the presence of two differently oriented muscle layers between the ribs. Indeed, the external intercostals have the same fiber orientation as the abdominal external oblique, while the internal intercostals are oriented in a parallel direction with the abdominal internal oblique. In the same way that these abdominal muscles are known to have important functions as rotators of the trunk, the primary purpose of the external and internal intercostals might be to rotate the trunk. Clearly, a single muscle layer between the ribs, no matter what its fiber orientation would be, or a simple elastic membrane would hinder such body movements. Only two muscle layers oriented obliquely at right angles to each other may permit the ribs to rotate relative to one another and with the vertebral column equally in two different directions.

\section{References}

1. Beau, J. H. S., and J. H. Maissiat. 1843. Recherches sur le mécanisme des mouvements respiratoires. Arch. Gen. Méd. 1:265-295.

2. Duchenne, G. B. 1867. Physiologie des mouvements. Baillière, Paris. 611-700.

3. Luciani, L. 1911. Circulation and respiration. In Human Physiology, Vol. 1. Macmillan Publishing Co., London. 411-415.

4. Gesell, R. 1936. Individuality of breathing. Am. J. Physiol. 115: 168-180.

5. Campbell, E. J. M. 1955. An electromyographic examination of the role of the intercostal muscles in breathing in man. J. Physiol. (Lond.) 129:12-26.

6. Hamberger, G. E. 1727. De Respirationis Mechanismo. Iena.

7. Bronk, D. W., and L. K. Ferguson. 1935. The nervous control of intercostal respiration. Am. J. Physiol. 110:700-707.

8. Taylor, A. 1960. The contribution of the intercostal muscles to the effort of respiration in man. J. Physiol. (Lond.) 151:390-402.

9. Draper, M. H., P. Ladefoged, and D. Whitteridge. 1960. Expiratory pressures and air flow during speech. Br. Med. J. 1:1837-1843.

10. Delhez, L. 1974. Contribution électromyographique à l'étude de la mécanique et du contrôle nerveux des mouvements respiratoires de l'homme. Vaillant-Carmanne Liège, Belgium.

11. De Troyer, A., and S. Kelly. 1982. Chest wall mechanics in dogs with acute diaphragm paralysis. J. Appl. Physiol. Respir. Environ. Exercise Physiol. 53:373-379.

12. De Troyer, A., S. Kelly, and W. A. Zin. 1983. Mechanical action of the intercostal muscles on the ribs. Science (Wash. DC). 220: 87-88.

13. Konno, K., and J. Mead. 1968. Static volume-pressure characteristics of the rib cage and abdomen. J. Appl. Physiol. 24:544-549.

14. Mellissimos, C. G., E. N. Bruce, M. D. Goldman, E. Elliott, and J. Mead. 1981. Pattern of diaphragmatic activity during forced expiratory vital capacity. J. Appl. Physiol.: Respirat. Environ. Exercise Physiol. 51:1515-1525.

15. Duron, B. 1981. Intercostal and diaphragmatic muscle endings and afferents. In Regulation of Breathing. Part I. T. F. Hornbein, editor. Marcel Dekker Inc., New York. 473-540.

16. Campbell, E. J. M. 1955. The role of the scalene and sternomastoid muscles in breathing in normal subjects: an electromyographic study. J. Anat. 89:378-386.

17. Raper, A. J., W. T. Thompson, Jr., W. Shapiro, and J. L. Patterson, Jr. 1966. Scalene and sternomastoid muscle function. $J$. Appl. Physiol. 21:497-502.

18. Druz, W. S., and J. T. Sharp. 1981. Activity of respiratory muscles in upright and recumbent humans. J. Appl. Physiol. Respir. Environ. Exercise Physiol. 51:1552-1561.

19. D'Angelo, E. 1982. Inspiratory muscle activity during rebreathing in intact and vagotomized rabbits. Respir. Physiol. 47:193-218.

20. Koepke, G. H., E. M. Smith, A. J. Murphy, and D. G. Dickinson. 1958. Sequence of action of the diaphragm and intercostal muscles during respiration. I. Inspiration. Arch. Phys. Med. Rehabil. 39:426-430.

21. Van Lunteren, E., M. A. Haxhim, N. S. Cherniack, and M. D. Goldman. 1983. Effects of posture and hypercapnia on chest wall muscle activity. Fed. Proc. 42:1012. (Abstr.)

22. Murphy, A. J., G. H. Koepke, E. M. Smith, and D. G. Dickinson. 1959. Sequence of action of the diaphragm and intercostal muscles during respiration. II. Expiration. Arch. Phys. Med. Rehabil. 40:337-342.

23. Jones, D. S., and J. E. Pauly. 1957. Further electromyographic studies on muscles of costal respiration in man. Anat. Rec. 128:733746. 